\title{
A New Preprocessing Method on Evidence Weight of Dempster Fusion Rule
}

\author{
Ying-chun $\mathrm{Li}^{1,}$, , Wei Xiong ${ }^{1, \mathrm{~b}}$, De-sheng $\mathrm{Liu}^{1, \mathrm{c}}$ and Jian-Bo Cheng ${ }^{1, \mathrm{~d}}$ \\ ${ }^{1}$ Science and Technology on Complex Electronic System Simulation Laboratory, Equipment \\ Academy, Beijing, 101400, China \\ aliyingchun1993@163.com, 'Weixiong@189.cn, ${ }^{\mathrm{c} l i u d s @ 263 . n e t, ~}{ }^{\mathrm{d}}$ 742297503@qq.com
}

Keywords: D-S evidence theory, Fusion rule, Belief mass, Evidence weight, Reassignment

\begin{abstract}
In allusion to the three problems of conventional D-S (Dempster-Shafer) evidence theory, this paper proposes an improved Dempster fusion rule based on weight preprocessing. By comparing the fusion results with conventional method and other improved methods, we conclude that the method in this paper is more superior in dealing with uncertainty, and more reasonable in processing the evidence weight.
\end{abstract}

\section{Introduction}

D-S evidence theory, also known as Dempster-Shafer theory or Belief theory, is the extension of classical probability theory. This theory was proposed by Dempster. In 1960s and supplemented by his student Shafer ${ }^{[1]}$. Because it does not need prior probability as a basis for reasoning, and can distinguish between "not certain” and “don't know”, D-S evidence theory has been widely applied in multi-source information fusion, artificial intelligence, system evaluation and network analysis ${ }^{[2]}$ etc.

However, the core fusion rule of conventional D-S evidence theory, Dempster fusion formula, has very large defects. For example, it will give counter-intuitive result when fusing high conflict information, and it has "one vote veto" problem and evidence weight problem etc. These problems affect the further promotion of D-S evidence theory. To expand the application of D-S evidence theory, this paper is going to propose a method to improve Dempster fusion rule to solve these problems and deal with evidence weights more reasonably.

\section{D-S evidence theory}

Introduction. In D-S evidence theory, a sample space is called a discernment framework, expressed as $\Theta=\left(\theta_{1}, \theta_{2} \ldots \theta_{n}\right)$. It is a finite nonempty set, and the elements in it are mutually exclusive. For each subset of $\Theta$, a probability can be assigned to it, which is called a BPA (Basic Probability Assignment). This probability assignment in D-S evidence theory can be assigned to evidence source which is like "mass" in physics, so it can be also called belief mass or mass function ${ }^{[3]}$.

Definition Set $\Theta$ is a discernment frame, mapping $m: 2^{\Theta} \rightarrow[0,1]$ is a mass function or a BPA of the one certain subset $A$ of $\Theta, A$ is called a focal element. Suppose if $A=\varnothing$ (Ø stands for empty set), $m(\varnothing)$ $=0$; if $\mathrm{A} \neq \varnothing$, then,

$$
\sum_{A \in 2^{\Theta}} m(A)=1
$$

The basic strategy of D-S evidence theory is to fuse the independent judgements on the discernment frame from different sides by Dempster fusion rule, in order to decrease the uncertainty of the judgements. The rule is as follows.

Fusion rule: set $m_{i}$ is a certain mass function on $\Theta$, the value of $m_{i}\left(A_{\mathrm{j}}\right)$ is the belief mass assigned on an evidence source $A_{\mathrm{j}}, \mathrm{j}=1,2 \ldots \mathrm{k} . \quad A_{\mathrm{j}}$ and $A$ are subsets of the discernment frame $\Theta$. Then the orthogonal sum $m=m_{1} \oplus m_{2} \ldots \oplus m_{n}$ is defined as, 


$$
m(A)=\left\{\begin{array}{c}
\frac{1}{1-k} \sum_{\cap^{A_{j}}=A} \prod_{i=1}^{n} m_{i}\left(A_{j}\right), A \neq \varnothing \\
0, A=\varnothing
\end{array}\right.
$$

And

$$
k=\sum_{\cap^{A_{j}}=\varnothing} \prod_{i=1}^{n} m_{i}\left(A_{j}\right)
$$

I the formula, $\mathrm{k}$ is called conflict factor, which stands for conflict level of all the evidences. $0<\mathrm{k}<1$, the bigger $\mathrm{k}$ is, the greater the conflict is, and vice versa.

Defects of Dempster fusion rule. As a method of uncertain knowledge reasoning, D-S evidence theory has been widely applied on uncertain information fusing and reasoning ${ }^{[4][5]}$. However, there are some limitations in the application of the conventional D-S evidence theory.

- Firstly, conventional Dempster fusion formula dose not distinguish every evidence but consider them as similar with the same weight. This is not practical especially in expert based systems, multi-source systems and so on. The weight of an evidence source determines how much we trust it, so that it should be considered whether it is provided or not.

- Secondly, the rule can only get better results when the conflict level of evidences is low, when it is high or completely contradictory, the fusion result will be often counter-intuitive. This is because that the formula abnegates all the conflict belief mass, which is be considered totally useless ${ }^{[6]}$.

- Thirdly, it has "one vote veto" problem which can be seen in the fusion formula, If one mass function of an evidence source is zero, i.e. $m\left(A_{\mathrm{j}}\right)=0$, then no matter how much the belief mass on $A_{\mathrm{j}}$ assigned by other evidence source, the final fusion result is always 0 , like "one vote veto" in the election.

\section{Representative improvement methods and their weight processing approaches}

There are two kinds of improvement about the fusion rule of D-S evidence theory, one is the improvement of the fusion formula, and the other is to preprocess the evidence sources ${ }^{[7]}$.

The first kind of improvement.The representatives of the first kind of improvement is Yager et al. They think the reason resulting in counter-intuitive outcome is that Dempster abnegated all the conflict information. This kind of belief mass should be assigned on uncertainty or other focal elements in proportion to the conflict factor. Yager threw all the belief onto uncertainty, and his new fusion formula is as follow,

$$
\left\{\begin{array}{c}
m(A)=\sum_{\cap^{A_{i}=A^{1}}} \prod_{j \leq n} m_{j}\left(A_{i}\right),(A \neq \varnothing, A \neq \Theta) \\
m(\Theta)=\sum_{\cap^{A_{i}=\Theta 1 \leq j \leq n}} \prod_{j} m_{j}\left(A_{i}\right)+k \\
m(\varnothing)=0
\end{array}\right.
$$

$k$ is the conflict factor.

$\mathrm{Li} \mathrm{Bi}$-cheng's method ${ }^{[8]}$, one kind of the modification of Yager's method, is a relatively mature one in the first kind of improvements. He thought that conflict information is not totally useless or uncertain, the belief on it should be reassigned. His new fusion formula is as follow,

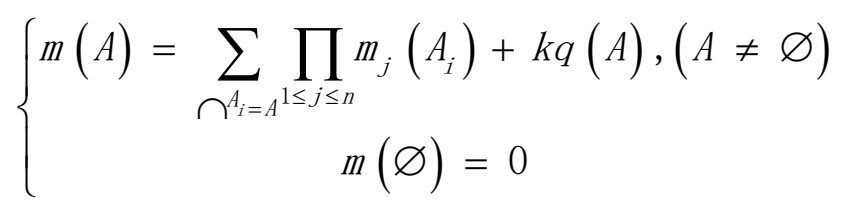

Among them, $k$ is the conflict factor, and $q$ is the average reliability of evidence source, 


$$
q(A)=\frac{1}{n} \sum_{i=1}^{n} m_{i}(A)
$$

It can be seen from the improved formula, Li removed the denominator to retain the belief mass on conflict information, and added a term $k q(\mathrm{~A})$ to avoid "one vote veto" issue. However, this kind of improvement doesn't consider the evidence weight problem. The formula still regards every evidence as the same.

The second kind of improvement. The representatives of the second kind of improvement is Murphy et al. They think that conventional Dempster fusion formula has strong math foundation, and it is easy to be applied on industry because it is fit to associative law. So we should improve the mathematic model rather than the formula. In order to alleviate the conflict of the evidence, Murphy's method $^{[9]}$ used simple average thinking to average the belief mass on one focal element from all the evidences, and then fuse them $n-1$ times by Dempster fusion rule( $n$ is the number of evidence). Deng Yong $^{[10]}$ modified Murphy method on mathematic, specifically, he changed Murphy's "average” to "weighted average". The weights are evidences' weight or expert authority in expert based system. Another preprocess method on evidence sources is called "Discount method" which is proposed by Yang Chun ${ }^{[11]}$. He discounted all the belief mass in proportion to the evidences' weight, and put the extra mass onto uncertainty. His idea is that if an evidence has a lower weight, it gives bigger uncertainty $^{[12]}$. The preprocess formula on evidence source is as follows,

$$
\left\{\begin{array}{c}
m_{i}^{\prime}\left(A_{j}\right)=\omega_{i} m_{i}\left(A_{j}\right) \\
m_{i}^{\prime}(\Theta)=\omega_{i} m_{i}(\Theta)+\left(1-\omega_{i}\right)
\end{array}\right.
$$

Because of the processing on the evidence sources, by weakening the conflict between the evidence, Deng Yong's method and "Discount method" solved the above three problems, and retained the advantage that conventional Dempster rule is easy to be applied on industry. However, on evidence weight problem, they also have shortcomings. Deng Yong inherited Murphy's average thinking and considered evidence weights, but this kind of thinking will completely lose the specific information and make all the evidence information tend to accordance. This can no doubt decrease uncertainty, but it doesn't accord with the truth. "Discount method" is a relatively conservative method which can be seen from the preprocess formula. It puts all the extra belief mass from focal elements' discount to uncertainty. This approach will increase uncertainty to a great extent.

This paper will propose a new evidence source reprocess method, and compare its result with $\mathrm{Li}$ Bi-cheng's method, Deng Yong's method and "Discount method".

\section{A new improvement method}

This paper agrees with that D-S evidence theory fusion rule has good mathematic foundation which should not be modified, so that we will use the second kinds of improvement, i.e. preprocessing evidence sources. Based on the principle that smaller weight of an evidence causes smaller effect on the fusion result, this paper proposed a new method to solve the high conflict problem and "one vote veto" problem. The method is going to reassign the belief mass according to evidence weight. In common sense, evidence weight determines the importance of the information no matter in expert based systems or other multi-sensor system. Evidence with smaller weight should give out more ambiguous opinions or information and vice versa. Suppose that if one evidence's weight is 1 , the belief mass should not be reassigned because the information is absolutely accurate, and if it's 0 , the information will be totally ambiguous, that is to say, the value of mass on every focal are the same (this is can be proved that if every belief mass of an evidence is the same, this evidence will not affect the fusion result.) So the improvement detail is:

- If $\omega=1$, no reassignment;

- If $\omega=0$, every belief mass will be the same, i.e. $m\left(A_{j}\right)=1 / n(n$ is the number of focal elements); 
- If $0<\omega<1$, In the process of varying from 1 to 0 , the belief mass changes simply linearly from the initial value to $1 / n$, ( $n$ is the number of sources of evidence).

So the preprocessing formula is,

$$
m^{\prime}\left(x_{i}\right)=\omega_{i} m\left(x_{i}\right)+\frac{1-\omega_{i}}{n}
$$

\section{Numerical example and comparisons}

Numerical example.Suppose there are three evidence sources, $a_{1}, a_{2}, a_{3}$ respectively. Their weights was calculate by evidence distance approach proposed by Jousselme ${ }^{[13]}$. Since this is not the focus of this paper, here is no detailed procedure, $\omega=(0.2,0.5,0.3)$. There are 6 focal elements in total, $A_{1}, A_{2}$, $A_{3}, A_{4}, A_{5}$ and $\Theta(\Theta$ means uncertainty). The belief mass matrix is as follows,

$$
R(X)=\left[\begin{array}{lll}
m_{1}\left(A_{1}\right) & m_{2}\left(A_{1}\right) & m_{3}\left(A_{1}\right) \\
m_{1}\left(A_{2}\right) & m_{2}\left(A_{2}\right) & m_{3}\left(A_{2}\right) \\
m_{1}\left(A_{3}\right) & m_{2}\left(A_{3}\right) & m_{3}\left(A_{3}\right) \\
m_{1}\left(A_{4}\right) & m_{2}\left(A_{4}\right) & m_{3}\left(A_{4}\right) \\
m_{1}\left(A_{5}\right) & m_{2}\left(A_{5}\right) & m_{3}\left(A_{5}\right) \\
m_{1}(\Theta) & m_{2}(\Theta) & m_{3}(\Theta)
\end{array}\right]=\left[\begin{array}{lll}
0.05 & 0.10 & 0.20 \\
0.05 & 0.25 & 0.25 \\
0.10 & 0.25 & 0.20 \\
0.40 & 0.00 & 0.10 \\
0.30 & 0.00 & 0.05 \\
0.10 & 0.40 & 0.20
\end{array}\right]
$$

By preprocessing with formula (8), we get,

$$
R^{\prime}(X)=\left[\begin{array}{lll}
0.14 & 0.13 & 0.18 \\
0.14 & 0.21 & 0.19 \\
0.15 & 0.21 & 0.18 \\
0.21 & 0.08 & 0.15 \\
0.19 & 0.08 & 0.13 \\
0.15 & 0.28 & 0.18
\end{array}\right]
$$

Put this matrix into conventional Dempster fusion rule, we can get the final result, $M(X)=(0.178$, 0.227, 0.225, 0.177, 0.156, 0.038).

Comparisons.As shown in Table 1 is the fusion results of conventional Dempster fusion rule, three representative improved D-S evidence theory methods and the new improved method in this paper. It can be seen that method in this paper can decrease the uncertainty efficiently and properly deal with evidence source weight. Li Bi-cheng's method and "Discount method" reassign the belief mass to uncertainty item before fusion, so that uncertainty of the result still remains highly uncertainty, information precision is still low. The result of Deng Yong's method has very low uncertainty, but it loses the unique information which will cause inaccuracy.

Table 1 The fusion results of different methods

\begin{tabular}{ccccccc}
\hline & $A_{1}$ & $A_{2}$ & $A_{3}$ & $A_{4}$ & $A_{5}$ & $\Theta$ \\
\hline $\begin{array}{c}\text { Conventional } \\
\text { Dempster rule }\end{array}$ & 0.113 & 0.185 & 0.227 & 0.268 & 0.165 & 0.041 \\
$\begin{array}{c}\text { Li Bi-cheng's } \\
\text { method }\end{array}$ & 0.116 & 0.184 & 0.192 & 0.186 & 0.126 & 0.196 \\
$\begin{array}{c}\text { Deng Yong's } \\
\text { method }\end{array}$ & 0.135 & 0.307 & 0.295 & 0.120 & 0.073 & 0.070 \\
$\begin{array}{c}\text { Discount } \\
\text { method }\end{array}$ & 0.130 & 0.256 & 0.249 & 0.087 & 0.057 & 0.221 \\
$\begin{array}{c}\text { Method in this } \\
\text { paper }\end{array}$ & 0.178 & 0.227 & 0.225 & 0.177 & 0.156 & 0.038 \\
\hline
\end{tabular}




\section{Conclusion}

By comparing the new improvement method with other methods, we get the following conclusions.

- This method can solve the three problems mentioned above in the D-S evidence theory. And through the fusion of information, uncertainty can be reduced.

- The new method adopts the method of preprocessing evidence sources and retains the original fusion formula, which doesn't weak the application of D-S evidence theory in industry.

- Compared with other methods, the new method is more reasonable to deal with the problem of evidence weight.

\section{References}

[1] Shafer G. A mathematical theory of evidence [M]. 1976.

[2] Zhangyang Qu, Yaying Li. A Network Security Situation Evaluation Method Based On D-S Evidence Theory[C] Environmental Science and Information Application Technology (ESIAT), 2010 International Conference. 2010:496-499.

[3] Dempster A P. Upper and Lower Probabilities Induced by a Multivalued Mapping [J]. Annals of Mathematical Statistics, 1967, 38(2):325-339.

[4] Chundong Wang, Yu Zhang. Network Security Situation Evaluation Based on Modified D-S Evidence Theory [J]. Wuhan University Journal of Natural Sciences, 2014, 19(5):409-416.

[5] Yibo Li, Ning Wang, et al. Based on D-S evidence theory of information fusion improved method[C] International Conference on Computer Application and System Modeling. IEEE, 2010:V1-416-V1-419.

[6] Xianfeng Fan, Ming J. Zuo. Fault diagnosis of machines based on D-S evidence theory. Part 1: D-S evidence theory and its improvement [J]. Pattern Recognition Letters, 2006, 27(5):366-376.

[7] Rui Sun, Hongzhong Huang, Qiang Miao. Improved information fusion approach based on D-S evidence theory [J]. Journal of Mechanical Science and Technology, 2008, 22(12):2417-2425.

[8] B Li, B Wang, J Wei, et al. Efficient combination rule of evidence theory [J]. Journal of Data Acquisition \& Processing, 2002, 17(1):237-240. (In Chinese)

[9] Murphy C K. Combining belief functions when evidence conflicts [J]. Decision Support Systems, 2000, 29(1):1-9.

[10]Yong Deng, Wengkang Shi, et al. Efficient combination approach of conflict evidence [J]. Journal of Infrared \& Millimeter Waves, 2004, 23(1):27-32. (In Chinese)

[11]Chun Yang, Huaizu Li. An Evidence Reasoning Model with Its Application to Expert Opinions Combination [J]. Systems Engineering theory \& Practice, 2001, 21(4):43-48. (In Chinese)

[12]Jianbo Yang, Xu D L. On the evidential reasoning algorithm for multiple attribute decision analysis under uncertainty [J]. Systems Man \& Cybernetics Part A Systems \& Humans IEEE Transactions on, 2002, 32(3):289-304.

[13]Jousselme A L, Grenier D, Éloi Bossé. A new distance between two bodies of evidence [J]. Information Fusion, 2001, 2(2):91-101. 